The theory of fluctuation conductivity for an arbitrary impurity concentration including ultraclean limit $\left(T \tau \gg \sqrt{\frac{T_{c}}{T-T_{c}}}\right)$ is developed. It is demonstrated that the formal divergency of the fluctuation density of states contribution obtained previously for the clean case is removed by the correct treatment of the non-local ballistic electron scattering. We show that in the ultra-clean limit the density-of-states quantum corrections are canceled by the Maki-Thompson term and only classical paraconductivity remains.

\title{
Strong compensation of the quantum fluctuation corrections in clean superconductor
} D.V.Livanov, G.Savona

Department of Theoretical Physics, Moscow State Institute of Steel and Alloys, Leninski pr. 4, Moscow 117936, Russia

A.A.Varlamov

Istituto Nazionale di Fisica della Materia(INFM)-Unità "Tor Vergata", Dipartimento di Scienze e Tecnologie Fisiche ed Energetiche, Università di Roma "Tor Vergata", via di Tor Vergata, 00133 Roma, Italy

PACS: $74.40 .+\mathrm{k}, 74.50 .+\mathrm{r}, 74.20 .-\mathrm{z}$

As it is well known, the first order fluctuation corrections to conductivity in the vicinity of superconducting transition are presented by the Aslamazov-Larkin (AL), Maki-Thompson (MT) and density of states (DOS) contributions. First one has the simple physical meaning of the direct charge transfer by the fluctuation pairs themselves and can be easily derived from the phenomenological time dependent Ginzburg-Landau equation [1]. In this sense it is a result characteristic for the classical electron theory, while Maki-Thompson and DOS contributions have the purely quantum origin and can be calculated in the frameworks of the microscopic approach only [2].

The character of the electron scattering plays a very special role for the manifestation of fluctuation effects. In the BCS theory of superconducting alloys the only criterion of the metal purity exists: it is the ratio between the Cooper pair "size" (zero temperature coherence length of pure metal $\xi_{0}$ ) and the electron mean free path $\ell$. If the alloy is dilute $\left(\ell \gg \xi_{0}\right)$ the Cooper pairs motion is ballistic and impurities do not manifest themselves in superconductor properties. In the opposite case $\ell \ll \xi_{0}$ the Cooper pairs motion has the diffusive character and the role of the effective Cooper pair size plays the renormalized coherence length $\xi^{\prime}=\sqrt{\ell \xi_{0}}$. The relative magnitude of fluctuation effects, which is determined by the Ginzburg-Levanyuk number, is proportional to $(a / \xi)^{n}$ ( $a$ is an interatomic distance and $n>0$ depends on the effective dimensionality of the electron spectrum) and it grows for impure systems.

Dealing with the superconductor electrodynamics in fluctuation regime it is necessary to remember that in the vicinity of the critical temperature the role of fluctuation Cooper pair effective size plays the Ginzburg-Landau coherence length $\xi_{G L}(T)=\xi_{0} / \sqrt{\varepsilon}$ (where the reduced temperature $\varepsilon=\left(T-T_{c}\right) / T_{c}$ ). So the case of dilute metal $\left(\ell \gg \xi_{0}\right)$ in the vicinity of the transition could be formally subdivided on clean, which is still local $\left(\xi_{0} \ll \ell \ll \xi_{G L}(T)\right)$ and ultra-clean, non-local $\left(\xi_{G L}(T) \ll \ell\right)$ limits. In terms of the used in the theory of disordered alloys parameter $T \tau$ the same three domains can be written down as $T \tau \ll 1 ; 1 \ll T \tau \ll 1 / \sqrt{\varepsilon}$ and $1 / \sqrt{\varepsilon} \ll T \tau$. (We use units $k_{B}=\hbar=c=1$ ). The latter case was rarely discussed in literature [3 [5] in spite of the fact that it becomes of the first importance already for metals of very modest purity, let us say, $T \tau \approx 10$. Really, in this case the condition $T \tau \geq 1 / \sqrt{\varepsilon}$ read for the reduced temperature as $10^{-2} \leq \varepsilon \ll 1$ practically covers all experimentally accessible range of temperatures for the fluctuation conductivity measurements. What concerns the usually considered local clean case $(1 \ll T \tau \ll 1 / \sqrt{\varepsilon})$ for chosen value $T \tau \approx 10$ it would not have any range of applicability: indeed, the equivalent condition for the allowed temperature interval $\varepsilon \ll 1 /(T \tau)^{2}$ almost contradicts to the $2 D$ thermodynamical GinzburgLevanyuk criterion of the mean field approximation applicability $\left(G i \sim \frac{T_{c}}{E_{F}} \ll \varepsilon\right)$. Moreover, as it is well known, for transport coefficients the high order corrections become to be comparable with the mean field results much before than for thermodynamical ones, namely at $\varepsilon \sim \sqrt{G i}[6,7]$. So in practice one can speak about the dirty, intermediate or ultra-clean cases but not about the clean one.

We will restrict our consideration by the most interesting case of $2 \mathrm{D}$ electron spectrum, relevant to the high temperature superconductors. As it is known, the classic 2D AL contribution turns out to be independent on the electron mean free path $\ell$ at all [B]:

$$
\delta \sigma_{A L}^{(2)}=\frac{e^{2}}{16} \frac{1}{\varepsilon}
$$


Anomalous Maki-Thompson contribution, being induced by the pairing on the Brownian diffusive trajectories [2], naturally depends on $T \tau$, but its form changes only when $\ell \sim \xi_{G L}(T)(T \tau \sim 1 / \sqrt{\varepsilon})$. Its calculation, even with the non-local Cooperon vertices but the standard propagator, in the ultra-clean limit [4] leads to the expression less divergent in its temperature dependence but growing as $T \tau \ln (T \tau)$ with the increase of $\ell$ [3, 4]

$$
\sigma_{M T(a n)}^{(2)}=\frac{e^{2}}{8}\left\{\begin{array}{c}
\frac{1}{\varepsilon-\gamma_{\varphi}} \ln \left(\varepsilon / \gamma_{\varphi}\right), T_{c} \tau \ll 1 / \sqrt{\varepsilon} \\
\frac{8 \pi^{2} T \tau}{\sqrt{14 \zeta(3)}} \frac{1}{\sqrt{\varepsilon}} \ln \left(T_{c} \tau \sqrt{\varepsilon}\right), 1 / \sqrt{\varepsilon} \ll T_{c} \tau
\end{array}\right.
$$

where $\gamma_{\phi}=\pi / 8 T \tau_{\phi}$ is the inelastic scattering rate.

The analogous problem takes place in the DOS and regular part of MT contributions: their standard diagrammatic technique calculations lead to the negative correction [9]

$$
\begin{gathered}
\sigma_{D O S+M T(\text { reg })}^{(2)}=-\frac{e^{2}}{4 s} \kappa(T \tau) \ln \left(\frac{1}{\varepsilon}\right) \\
\kappa(T \tau)=\frac{-\psi^{\prime}\left(\frac{1}{2}+\frac{1}{4 \pi \tau T}\right)+\frac{1}{2 \pi \tau T} \psi^{\prime \prime}\left(\frac{1}{2}\right)}{\pi^{2}\left[\psi\left(\frac{1}{2}+\frac{1}{4 \pi \tau T}\right)-\psi\left(\frac{1}{2}\right)-\frac{1}{4 \pi \tau T} \psi^{\prime}\left(\frac{1}{2}\right)\right]}=\left\{\begin{array}{c}
\frac{56 \zeta(3)}{\pi^{4}} \approx 0.691, T \tau \ll 1 \\
\frac{8 \pi^{2}}{7 \zeta(3)}(T \tau)^{2}, T \tau \gg 1
\end{array}\right.
\end{gathered}
$$

evidently divergent when $T \tau \rightarrow \infty$.

In the derivation of these results the local form of the fluctuation propagator and Cooperons (besides (2)) were used. It is why in view of the mentioned above peculiarity of ultra-clean limit, the extension of their validity for $T \tau \gg 1 / \sqrt{\varepsilon} \rightarrow \infty$ seems to be doubtful.

One can notice that at the upper limit of the clean case, when $T \tau \sim 1 / \sqrt{\varepsilon}$ both DOS and anomalous MT contributions turn out to be of the same order of value but of the opposite signs. So one can suspect that in the case of correct procedure of the impurities averaging in the ultra-clean case the large negative DOS contribution can be cancelled with the positive anomalous MT one.

The reexamination of all fluctuation corrections of the first order in the case of the arbitrary impurity concentration including non-local electron scattering in the ultra-clean superconductor will be the aim of this communication. The nontrivial cancellation of the contributions, previously divergent in $T \tau$, will be shown. It results in the reduction of the total fluctuation correction in ultra-clean case to the AL term only.

In purpose to calculate the Cooperon (impurity vertex) $C\left(\mathbf{q}, \epsilon_{1}, \epsilon_{2}\right)$ and fluctuation propagator $L\left(\mathbf{q}, \omega_{\mu}\right)($ the twoparticle Green function) in the general case of an arbitrary electron mean free path case one needs the explicit expression for the polarization operator $\mathcal{P}\left(\mathbf{q}, \epsilon_{1}, \epsilon_{2}\right)$, which due to the elasticity of scattering does not contain the frequency summation and for $2 D$ spectrum has a form:

$$
\mathcal{P}\left(\mathbf{q}, \epsilon_{1}, \epsilon_{2}\right)=\int \frac{d^{2} p}{(2 \pi)^{2}} G\left(\mathbf{p}+\mathbf{q}, \epsilon_{1}\right) G\left(-\mathbf{p}, \epsilon_{2}\right)=\frac{2 \pi N(0) \Theta\left(-\epsilon_{1} \epsilon_{2}\right)}{\sqrt{v_{F}^{2} q^{2}+\left(\widetilde{\epsilon}_{1}-\widetilde{\epsilon}_{2}\right)^{2}}}
$$

where $\Theta(-x)$ is the Heaviside theta-function, $\widetilde{\epsilon}_{n}=\epsilon_{n}+\frac{1}{2 \tau} \operatorname{sgn} \epsilon_{n}, N(0)$ and $v_{F}$ are the density of states and the velocity at the Fermi level. Let us stress that this result was carried out without any expansion over the Cooper pair center of mass momentum $\mathbf{q}$ and is valid for an arbitrary $\ell q$.

The standard ladder consideration results in the following expressions for the Cooperon and fluctuation propagator:

$$
C^{-1}\left(\mathbf{q}, \epsilon_{1}, \epsilon_{2}\right)=1-\frac{\Theta\left(-\epsilon_{1} \epsilon_{2}\right)}{\tau \sqrt{v_{F}^{2} q^{2}+\left(\widetilde{\epsilon}_{1}-\widetilde{\epsilon}_{2}\right)^{2}}}
$$

and

$$
\begin{aligned}
-\left[N(0) L\left(\mathbf{q}, \Omega_{\mu}\right)\right]^{-1}= & \ln \frac{T}{T_{c}}+\sum_{n=0}^{\infty}\left\{\frac{1}{n+1 / 2}\right. \\
& \left.-\frac{1}{\sqrt{\left(n+\frac{1}{2}+\frac{\Omega_{\mu}}{4 \pi T}+\frac{1}{4 \pi T \tau}\right)^{2}+\frac{v_{F}^{2} \mathbf{q}^{2}}{16 \pi^{2} T^{2}}}-\frac{1}{4 \pi T \tau}}\right\}
\end{aligned}
$$


Near $T_{c} \ln \frac{T}{T_{c}} \approx \varepsilon$ and for the local limit, when just small momenta $\ell q \ll 1$ are involved in the final integrations, the Eqs. (6) and (7) can be expanded over $v_{F} q / \max \left\{T, \tau^{-1}\right\}$ and they are reduced to the well known local expressions.

The Feynman diagrams which contribute to conductivity in the first order of perturbation theory on electronelectron interaction in Cooper channel are presented in Fig.1. Let us start from the discussion of the Maki-Thompson contribution (diagram 6). We restrict our consideration by the vicinity of the critical temperature, where, for the most singular in reduced temperature contribution, static approximation is valid. It means that Cooper pair bosonic frequency can be assumed $\Omega_{\mu}=0$.

Using the general expressions for Cooperons and propagator (6), (7) after the integration over electronic momentum, one can find:

$$
\begin{aligned}
Q^{(6)}\left(\omega_{v}\right)= & -4 \pi N(0) v_{F}^{2} e^{2} T^{2} \sum_{\varepsilon_{n}} \int \frac{d^{2} \mathbf{q}}{(2 \pi)^{2}} L(\mathbf{q}, 0) \times \\
& {\left[\mathcal{M}\left(\tilde{\epsilon}_{n}, \tilde{\epsilon}_{n+\nu}, \mathbf{q}\right)+\mathcal{M}\left(\tilde{\epsilon}_{n+\nu}, \tilde{\epsilon}_{n}, \mathbf{q}\right)\right], }
\end{aligned}
$$

where

$$
\mathcal{M}(\alpha, \beta, \mathbf{q})=\frac{R_{q}(2 \alpha) R_{q}(\alpha+\beta)-\Theta(\alpha \beta) R_{q}(2 \alpha) R_{q}(2 \beta)}{(\beta-\alpha)^{2}\left(R_{q}(2 \alpha)-\frac{1}{\tau}\right)\left(R_{q}(2 \beta)-\frac{1}{\tau}\right) R_{q}(\alpha+\beta)}
$$

$R_{q}(x)=\sqrt{x^{2}+v_{F}^{2} \mathbf{q}^{2}}$.

The analogous consideration of the main in the clean case DOS diagrams 2 and 4 leads to:

$$
\begin{array}{r}
Q^{(2+4)}\left(\omega_{v}\right)=4 \pi N(0) v_{F}^{2} e^{2} T^{2} \sum_{\varepsilon_{n}} \int \frac{d^{2} \mathbf{q}}{(2 \pi)^{2}} L(\mathbf{q}, 0) \times \\
{\left[\mathcal{D}\left(\tilde{\epsilon}_{n}, \tilde{\epsilon}_{n+\nu}, \mathbf{q}\right)+\mathcal{D}\left(\tilde{\epsilon}_{n+\nu}, \tilde{\epsilon}_{n}, \mathbf{q}\right)\right],}
\end{array}
$$

with

$$
\begin{aligned}
\mathcal{D}(\alpha, \beta, \mathbf{q})= & (\beta-\alpha)^{2}\left(R_{q}(2 \alpha)-\frac{1}{\tau}\right)^{2} \times \\
& {\left[\frac{R_{q}^{2}(2 \alpha)+2 \alpha(\alpha-\beta)}{R_{q}(2 \alpha)}-\frac{\Theta(\alpha \beta) R_{q}^{2}(2 \alpha)}{\left(R_{q}(\alpha+\beta)-\frac{1}{\tau}\right)}\right] }
\end{aligned}
$$

One can see that each of expressions for $Q^{(6)}\left(\omega_{v}\right)$ and $Q^{(2+4)}\left(\omega_{v}\right)$, in accordance with [9] 10], in the limit $T \tau \rightarrow \infty$ presents itself the Loran series of the type $C_{-2}(T \tau)^{2}+C_{-1}(T \tau)+C_{0}+C_{1}(T \tau)^{-1}+\ldots$. The careful expansion of the sum of expressions (8) and (10) in the series of such type leads to the exact cancellation of all divergent contributions and even to the coefficient $C_{0}=0$. In result, the leading order of the sum of MT and DOS contributions in the limit of $T \tau \gg 1$ turns out to be $C_{1}(T \tau)^{-1}$ and it disappears in the non-local limit. The results of numerical calculation of $Q^{(6)}\left(\omega_{v}\right)+Q^{(2+4)}\left(\omega_{v}\right)$ as the function of $T \tau$ according to Eqs. (8) and (10) are presented in Fig. 2 for different temperatures. One can convince himself in the rapid decrease of this sum with the increase of $T \tau$.

The remained four diagrams among the first order fluctuation corrections to conductivity (see, for example, the Fig.9 in the review article [2]) are negligible in the vicinity of $T_{c}$. The similar consideration of the remaining two DOS-like diagrams (3) and (5) gives

$$
\begin{aligned}
Q^{(3+5)}\left(\omega_{v}\right)= & 4 \pi N(0) v_{F}^{2} e^{2} T^{2} \sum_{\varepsilon_{n}} \int d^{2} \mathbf{q} L(\mathbf{q}, 0) \times \\
& {\left[\mathcal{K}\left(\tilde{\epsilon}_{n}, \tilde{\epsilon}_{n+\nu}, \mathbf{q}\right)+\mathcal{K}\left(\tilde{\epsilon}_{n+\nu}, \tilde{\epsilon}_{n}, \mathbf{q}\right)\right], }
\end{aligned}
$$

where

$$
\mathcal{K}(\alpha, \beta, \mathbf{q})=\frac{2 \beta \Theta(-\alpha \beta)}{(\beta-\alpha)\left(R_{q}(2 \alpha)-\frac{1}{\tau}\right)^{2} R_{q}(2 \alpha)}
$$

Evaluation of Eq.(11) demonstrates that for $T \tau \gg 1$ the final contribution of diagrams 3 and 5 does not contain $\tau$-dependence, and is less $(\propto \ln 1 / \varepsilon)$ singular if compared with paraconductivity, in spite of the fact that each of diagrams 3 and 5 contains the divergent Loran term $\propto T \tau$ which cancel each other. 
So one can see that the DOS term divergence $\propto(T \tau)^{2}$, found before for clean case [9,10], has a restricted validity and can not be extended to $T \tau \rightarrow \infty$. In the limit of defectless superconductor the total DOS+MT contribution is proportional to $\ln 1 / \varepsilon$ and is independent on $T \tau$.

Finally let us turn to the discussion of the AL contribution. In this case, as it is well known, even in the vicinity of $T_{c}$ we cannot restrict ourselves by the static approximation and analytical continuation over the external frequency has to be accomplished. The diagram 1 at Fig.1 represents the Aslamazov-Larkin contribution:

$$
Q^{A L}\left(\omega_{v}\right)=\frac{e^{2}}{2 \pi i} \int \frac{d^{2} \mathbf{q}}{(2 \pi)^{2}} \oint d z \operatorname{coth} \frac{z}{2 T} B^{2}\left(\mathbf{q}, z, \omega_{v}\right) L(\mathbf{q},-i z) L\left(\mathbf{q},-i z+\omega_{v}\right),
$$

where the three Green function blocks have to be calculated with the non-local Cooperons and the expression for the non-local propagators have to be used. This program is hard to be realized in the general form and at present has been tried to be solved with different approximations in the set of papers 11, 13, 12, 14. We are interested here to study the effect of non-locality on the AL contribution in the first hand sacrificing the $a c$ effects $(\omega \neq 0)$ and being in the vicinity of the transition $\varepsilon \ll 1$.. So we omit the $z, \omega_{\nu}$ dependence of the Green functions block

$$
B\left(\mathbf{q}, z=0, \omega_{v}=0\right)=-4 \pi N(0) T v_{F}^{2} q \sum_{n=0}^{\infty} \frac{1}{\left(\sqrt{4{\widetilde{\epsilon_{n}}}^{2}+v_{F}^{2} \mathbf{q}^{2}}-\frac{1}{\tau}\right)^{2}\left(\sqrt{4{\widetilde{\epsilon_{n}}}^{2}+v_{F}^{2} \mathbf{q}^{2}}\right)}
$$

and evaluate the AL contribution in this approximation numerically. These calculations show that the temperature dependence of paraconductivity turns out to be close to the classical $2 \mathrm{D} \varepsilon^{-1}$ regime. It is necessary to mention that relatively far from the transition, where our approximation strictly speaking is already not applicable, the calculated curve lies somewhat lower of the AL theory prediction in accordance with the short wave-length fluctuation calculations and experimental findings [2]. We note, that although Eqs. (13), (14) contain dependence on $\tau$, the paraconductivity is turned out to be $\tau$-independent in the entire range of parameter $T \tau$, analogous to the local $2 \mathrm{D}$ result (11).

Let us discuss the results obtained. First of all it is necessary to stress the observed strong cancellation of the DOS and MT contributions, which were found previously, within the local fluctuation theory, to be divergent in the limit $\tau \rightarrow \infty$ [9.10]. As we have demonstrated, the correct account of non-local scattering processes in the ultra-clean limit results in the impurity independent, logarithmic in reduced temperature, contribution negligible in comparison with the more singular AL one.

Let us remind that the fluctuation conductivity in the limiting case $\tau=\infty$ and for the non-zero frequency of the external electromagnetic field was studied in Ref. [11], where the similar problem of the $\omega^{-2}$-divergence( instead of $\tau^{2}$ in our case) of the contributions from different DOS and MT-like diagrams aroused. The sum of all relevant diagrams nevertheless was found to be regular and proportional to $\omega$. Moreover, the sum of all DOS and MT diagrams (which correspond to diagrams 6, 2 and 4 in Fig. 1) was shown to be zero in the case $\tau=\infty$. In the current publication we have confirmed this statement studying the more general case $\omega \rightarrow 0, \tau \rightarrow \infty$ with $\omega \tau \rightarrow 0$ and convincing ourselves that for the one-electron type DOS and MT fluctuation processes the final result does not depend on the order of the $\lim _{\omega \rightarrow 0, \tau \rightarrow \infty}\left(\sigma^{D O S}+\sigma^{M T}\right)$ calculation. We have evaluated the explicit dependence of the overall fluctuation conductivity on the parameter $T \tau$ and have demonstrated its regular character.

What concerns the AL contribution, the careful investigation of its clean limit was done in [13] by means of the analysis of the paraconductivity diagram structure in the coordinate representation. It was shown that the electric field does not interact directly with the fluctuation Cooper pairs, but it produces the effect on the quasiparticles forming these pairs only. The characteristic time of the change of the quasiparticle state is of the order of $\tau$. Consequently the one-particle Drude type conductivity in ac field, as it is well known, has the first order pole. What concerns the AL paraconductivity, due to the above mentioned reasons, the pole in it was found to be of the second order [13]:

$$
\sigma_{A L}(\omega)=\frac{\sigma_{A L}^{\text {dirty }}}{(1-i \omega \tau)^{2}}
$$

In spite of this difference one can see that the AL conductivity, like, the Drude one, vanishes at $\omega \neq 0, \tau \rightarrow \infty$ because in the absence of impurities the interaction of electrons does not produce any effective force acting on the superconducting fluctuations, while the $d c$ paraconductivity conserves its usual $\tau$-independent form.

In the present paper we have approached to the same problem of the investigation of the AL contribution in clean metal studying the general non-local case in q-space and have shown the independence of the $d c$ paraconductivity on the material purity. 
It is necessary to stress that the non-local forms of the Cooperon and fluctuation propagator have to be accounted not only for the ultra-clean case but in every problem where the relatively large bosonic momenta are involved: account for the dynamical and short wavelength fluctuations beyond the vicinity of critical temperature, the effect of relatively strong magnetic fields on fluctuations and weak localization corrections etc. Recently such approach was developed in the set of studies of the DOS fluctuation effects [3,4, 12] and the efforts to apply it to the complete microscopic calculation of the magnetoconductivity for arbitrary temperatures and fields is undertaken in 14 .

Authors are grateful to J.Axnas and C.Castellani for valuable discussions. This work was accomplished in the frameworks of the INTAS Grant \# 96-0452. 


\section{Figure Captions}

Fig.1. Feynman diagrams for the leading-order contributions to fluctuation conductivity. Wavy lines are fluctuation propagators, thin solid lines with arrows are impurity-averaged normal-state Green's functions, shaded semicircles are vertex corrections arising from impurities, dashed lines with central crosses are additional impurity renormalizations and shaded rectangles are impurity ladders. Diagram 1 is the Aslamazov-Larkin term; diagrams 2-5 arise from the corrections to the normal state density of states in the presence of impurity scattering; diagram 6 is the Maki-Thompson term.

Fig. 2. The illustration of the decrease of the sum of DOS and MT contributions with the increase of the mean free path for the different values of reduced temperature: $\varepsilon=0.001 ; 0.01 ; 0.1 ; 0.2$.

[1] R.J.Tray, A.T. Dorsey, Phys.Rev., B 47, 2715 (1993).

[2] A.Varlamov, G.Balestrino, E.Milani, D.Livanov, Advances in Physics, 48, n.6, p.655-783 (1999).

[3] J.B.Bieri, K.Maki, Phys.Rev., B 42, 4854 (1990).

[4] M.Randeria, A.Varlamov, Phys.Rev., B 50, 10401 (1994).

[5] A.M.Rudin, I.L.Aleiner, L.I.Glazman, Phys. Rev. B 55, 1203 (1997).

[6] A.I.Larkin, Yu.N.Ovchinnikov, Journal of Low Temp.Phys. 16, 56 (1973)

[7] A.A.Varlamov, V.V.Dorin, Soviet JETP, 57, 1089 (1983).

[8] L.G.Aslamazov, A.I.Larkin Soviet Solid State Physics, 10, 1102 (1968)

[9] L. B. Ioffe, A. I. Larkin, A. A. Varlamov, L. Yu, Phys. Rev. B 47, 8936 (1993).

[10] V.V.Dorin, R.A.Klemm, A.A.Varlamov, A.I.Buzdin, D.V.Livanov, Phys. Rev. B 48, 12951 (1993).

[11] L. Reggiani, R. Vaglio, A. A. Varlamov. Phys. Rev. B 44, 9541 (1991).

[12] M.Eschrig, D.Rainer, Souls, Phys. Rev. B 59, 12095 (1999).

[13] A.G.Aronov, Hikami, A.I.Larkin, Phys. Rev. B 51, 3880 (1995).

[14] J.Axnas, O.Rapp, unpublished. 


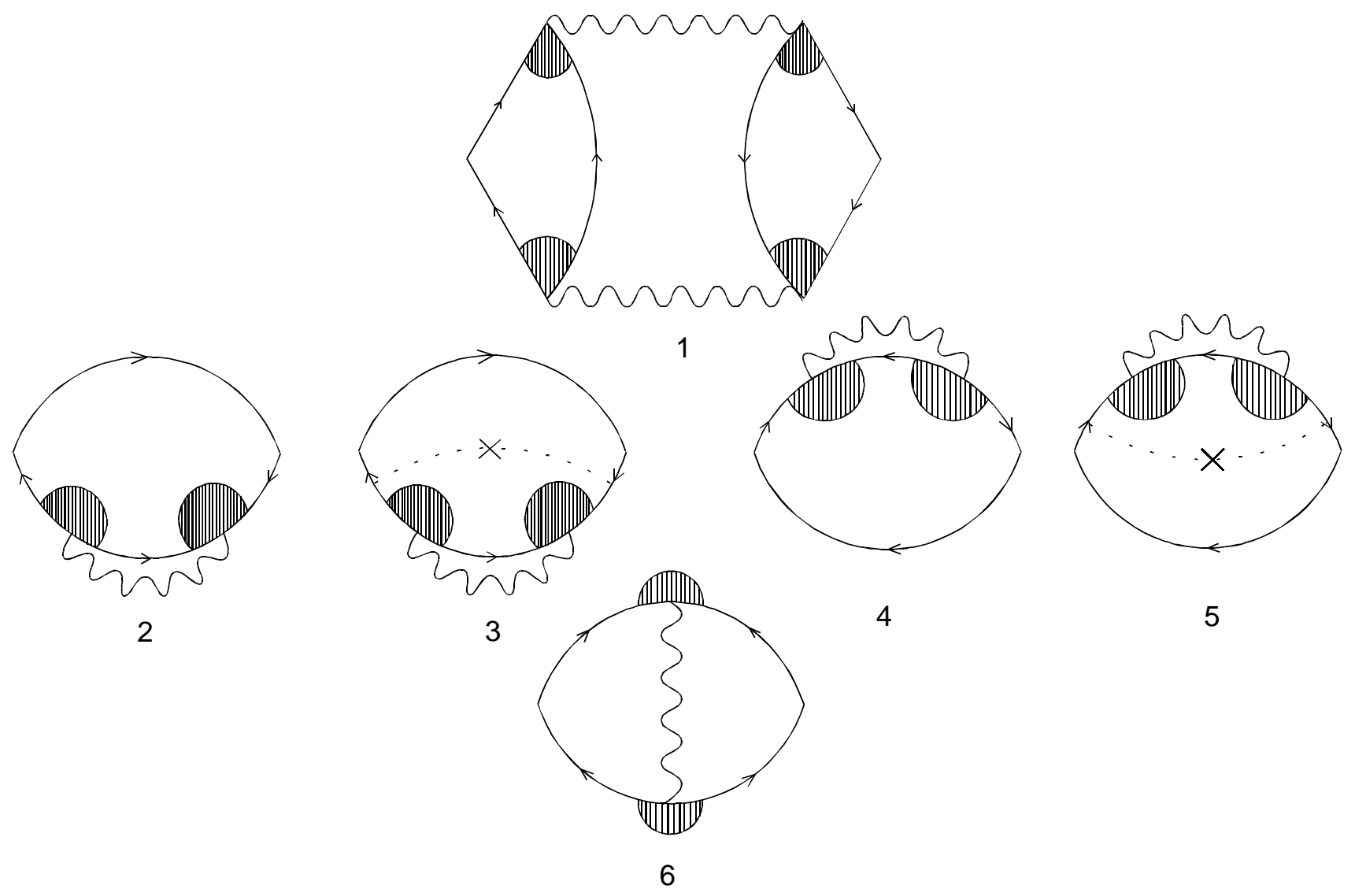




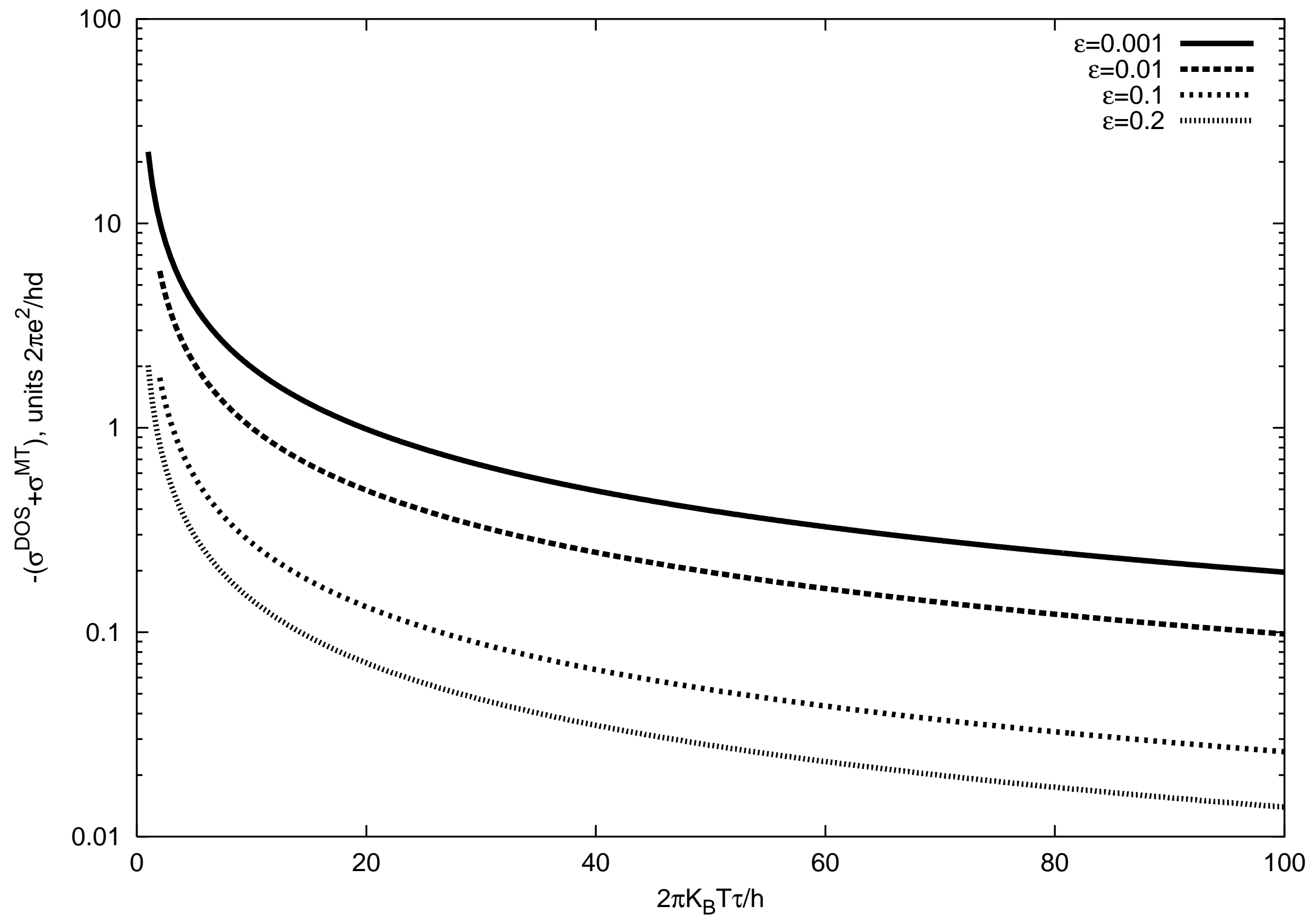

